\title{
Research and Implementation of the Low Voltage Power Line Communication System Based on OFDM
}

\author{
Rong Hua, Fuxiang Yi \\ School of Railway Transportation, Shanghai Institute of Technology, Shanghai, 201418, China
}

Keywords:Low Voltage Power Line Communication, OFDM.

\begin{abstract}
Low Voltage Power Line Communication (LVPLC) has entered the high-speed era, and it has got a lot of attention. Due to the huge power line network, there are not needed new lines for data transmission, so LVPLC system has a low cost and wide coverage advantages. The LVPLC system based on Orthogonal Frequency Division Multiplexing (OFDM) uses a plurality of mutually orthogonal sub-carriers for data transmission. It has high spectral efficiency and strong ability for anti-multipath interference.
\end{abstract}

\section{Introduction}

Low Voltage Power Line Communication is a new way that a relatively high frequency signal carrying the information is coupled to the power line, the power line as a medium for data transmission, and the high-frequency signal at the receiving end will be isolated from the power line and transfer to terminal apparatus [1]. "No New Wires" is the greatest advantages of LVPLC system [2]. In the low voltage power network, the low-speed LVPLC technology has first used in load control, remote meter reading and home automation.With the rapid development of high-speed PLC technology, In the low-voltage power line will likely simultaneous Internet access, voice, video, and power transmission.

This paper firstly analyzes the basic principle of low-voltage power line carrier communication system. Secondly, according to the low voltage power line channel environment is very poor, low voltage power line impedance, attenuation and noise characteristics need to be analyzed. Finally, analyze the OFDM technology principle and mathematical expressions, and realize the system simulation using MATLAB.

\section{The Basic Principle of Low Voltage Power Line Communication}

In our daily life, the power line is designed to output power of the media, and low voltage power lines are used to transmit $50 \mathrm{~Hz} / 220 \mathrm{~V}$ power frequency electrical energy, it is not a special signal transmission channel, as a signal transmission medium, we must first for data processing. According to the principle of the frequency shift and split, power line carrier technology will move the original signal to be sent to a different transmission frequency band, and through the coupling means coupling the signal to the power line.

Low voltage power line carrier communication system principle diagram as shown in figure.1. Signal from the source into the power line carrier modulation and demodulation unit 1 , coding and modulation of the signal, and processing it to the relatively high frequency signal, for the signal transmission in the low voltage power line. The processed signals are amplified by the power amplifying device to improve the distance and reliability of output signal in power line. then the signals are applied to low voltage power line through the coupling circuit.At the receiving end, Through the power line interface coupling circuit, we will put out the signal in the transmission of power line, and then through the power line modem unit 2 ,decoding and removing interference to get the original and useful signal to transmitted to the signal receiver. 


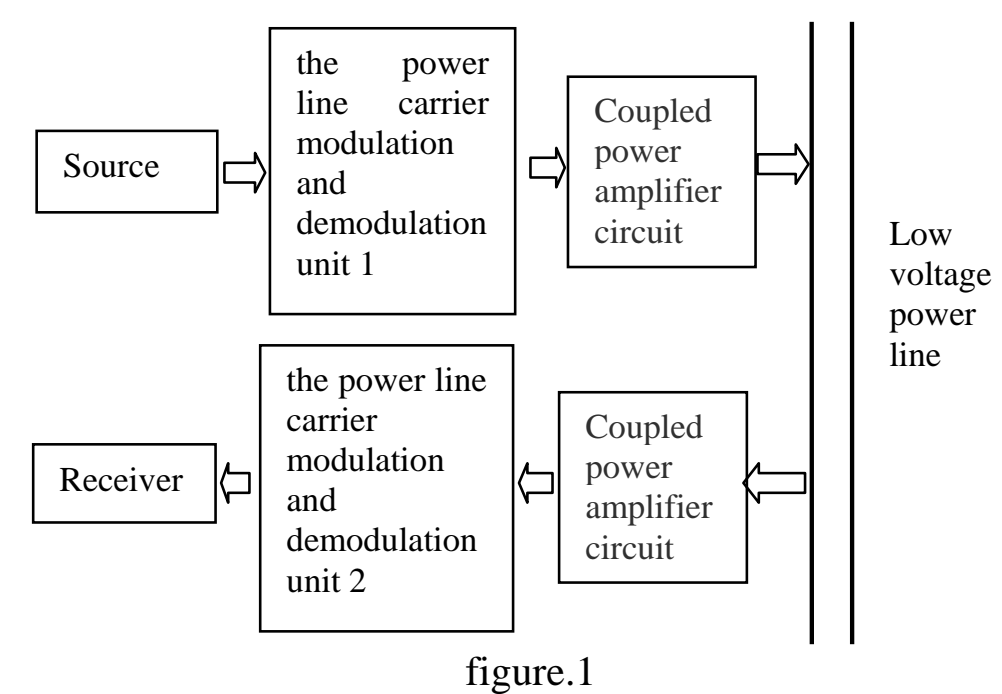

\section{Analysis of Low Voltage Power Line Channel Properties}

Input impedance, signal attenuation and anti-jamming performance of communication channel is the basic index to evaluate its performance in the field of Communication Science. Therefore it's necessary to study the several characteristics of low voltage power line.

\section{Impedance Characteristics.}

Impedance characteristics of the communication channel have a great influence on the distance and reliability of the signal transmission. The general low voltage power line distribution network terminal with rated frequency electric appliance is the basic $50 \mathrm{~Hz}$, therefore the low frequency impedance of the power line are relatively small. The input impedance of the low voltage power line value is low, and the overall performance of the impedance is increasing with frequency trend. Study on the correlation between the low pressure line input impedance and frequency of the transmission, the relationship between the input impedance and frequency for [3]:

$$
|z|=1.22 \times 10^{-2} \times f-0.06
$$

In the formula, the unit of frequency is $\mathrm{kHz}$; impedance unit is $\Omega$.

\section{Attenuation Characteristics.}

Low voltage power line signal attenuations are mainly coupling attenuation and line attenuation. Where the coupling attenuation occurs mainly in the coupling circuit terminal and the coupling attenuation is related to whether it matches with input impedance.Impedance of the power line itself is relatively stable, and does not arouse a significant attenuation of communication signal, the interference of the signal transmission is also very limited. But, when the low-voltage power line transmission frequency is much higher than the frequency of the high-frequency signal power, power lines are randomly distributed in the various properties of the load, making low-voltage power lines become a very non-uniform transmission medium. It will have a huge impact on transmitting of high-frequency signals.

i)Relationship between the signal attenuation and distance:In general, the farther the distance, the greater the signal attenuation. When the signal on the power line transmission, owing to the impedance mismatch, the signal will appear reflection, standing waves and other complex phenomena. This will make the relationship between the signal attenuation and transmission distance be complicated, not only shows that attenuation increases with distance, but also may be the close point attenuation greater than distant points.

ii)Relationship between the signal attenuation and frequency:The attenuation characteristics of the signals of different frequencies are completely different. In general, the higher the frequency, the greater the signal attenuation. In the low-voltage power line carrier communication system, when the signal transmission distance is short, it has little relationship between signal attenuation and carrier frequency. When the signal transmission distance is longer, the frequency is less than of $150 \mathrm{kHz}$ and the attenuation of the signal is still not great, while greater than $150 \mathrm{kHz}$, then there 
will be a lot of decay. Thus, the general set of low-voltage power line carrier communication system carrier frequencies below $150 \mathrm{kHz}$.

\section{Signal Attenuation Model.}

Build a short distance (about 30m) power line carrier systems combined with the above analysis, according to the signal transmission attenuation characteristic, signal attenuation model is available [4]:

$$
R(t)=c S(t)
$$

In the formula, $\mathrm{R}(\mathrm{t})$ is received signal; $\mathrm{S}(\mathrm{t})$ is transmission signal; $\mathrm{C}$ is signal attenuation coefficient.

From the foregoing, $-5 \mathrm{~dB}<20 \lg (\mathrm{R}(\mathrm{t}) / \mathrm{S}(\mathrm{t}))<0$, so $0.56<\mathrm{c}<1$.

\section{Noise Characteristics.}

Noises on the Low voltage power line can be simply divided into the following five categories:

i) colored background noise.

ii) narrow band noise.

iii) periodic pulse-frequency noise asynchronous with power Frequency.

iv) periodic pulse-frequency noise synchronous with power Frequency.

v) random pulse noise.

\section{Multipath Reflection Characteristics.}

In the signal transmission process, if the impedance of a network node mismatches with the signal, reflection will occur when the signal arrives at the network node. Signal reflection will change the original transmission path, thus there may be several paths to reach the receiver, resulting in signal arrival time and phase may be different.These reflected signals are superimposed and which may disturb the useful signal. And with the extension of the multipath delay time, the interference turns greater.

In the process of signal multipath propagation, the signal will also have a certain attenuation, the attenuation general performance flat fading and frequency selective fading. Among them, the flat fading slowly varying relative to the carrier frequency,is a flat attenuation, and it can be overcome by using OFDM technology.

\section{Establishment of Low Voltage Power Line Channel Model.}

This article selects $100 \mathrm{kHz}$ as a signal transmission frequency to establish a simplified model of low voltage power line channel. Signal transmission process by the noise, can be treated as additive random interference. Through the above research, the power line noises can be viewed as background noise superimposed on the other impulse noise. Figure.2 is the low voltage power line channel model.

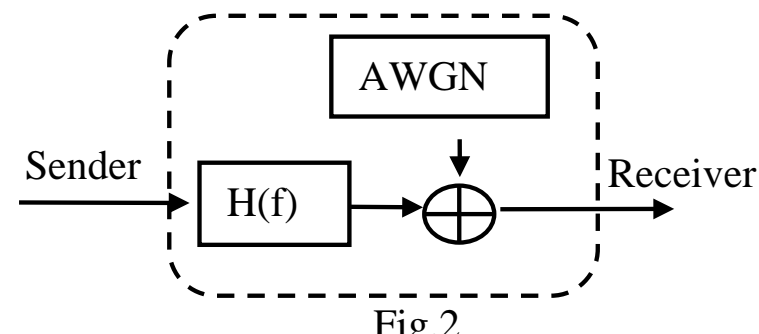

Low voltage power line power grid can be seen from the numerous T-shaped structures. Taking into account the relative relationship between attenuation and transfer function model of LVPLC system transmission distance power line signal can be written as [5]:

$$
H(f)=\sum_{i=1}^{N} g_{i} \exp \left(-\left(a_{0}+a_{0} f^{k}\right) d_{i}\right) \exp \left[-2 \pi \frac{d_{i}}{v_{p}}\right]
$$

Where: $\mathrm{i}$ is the path weight function; $\mathrm{k}$ is the decay parameter of the power line; the path length $\mathrm{i}$; $\mathrm{i}$ represents the delay path. 


\section{The Basic Principle of OFDM.}

The basic principle of OFDM is that coding the high speed information data ,and then assigning to $\mathrm{N}$ carriers which parallel and orthogonal to each other. The modulation rate on each carrier is very low $(1 / \mathrm{N})$. The continuous interval of modulation symbols is much larger than the time diffusion of channel.Thus it can provide effective protection for digital signal transmission in the environment which having a larger distortion and sudden pulse interference.OFDM uses the $\mathrm{N}$ overlapping sub bands, each sub channel baud rate is $1 / \mathrm{T}$, sub channel spacing is $1 / \mathrm{T}$, then the sub bands between each other are orthogonal.Therefore, the receiving end can receive data without the separation of spectrum.We can prove by IFFT that the orthogonal sub carrier modulation can be achieved.OFDM is not only a kind of modulation technology ,but also a kind of multiplexing technology.

The sender will transform the transmission digital data to mapping of the sub carrier amplitude and phase. And then,converting spectrum expression of the data into the time domain by IDFT transform. IFFT transform and IDFT transform has the same effect, just have a higher computational efficiency, so it can applicable to all application systems.The operation of the receiving end is opposed to the sending end.Mixing the RF signal and the base band signal,and then decomposing the frequency domain signal by FFT.The amplitude and phase of the sub carrier will be collected and converted back to digital signals.Figure.3 is a block diagram of the OFDM system[6].

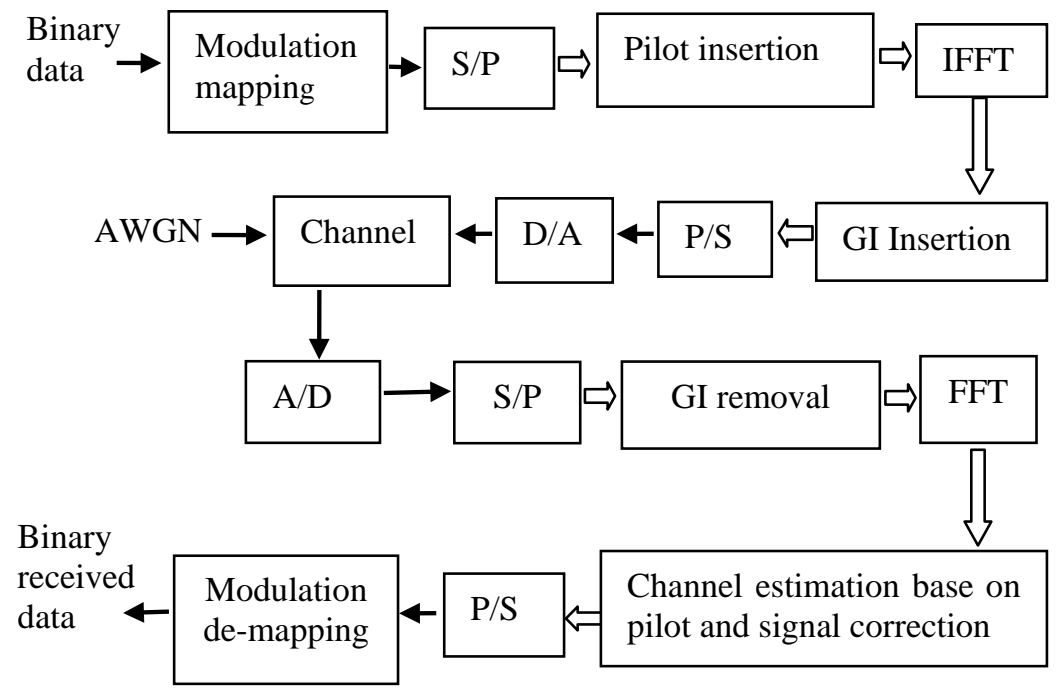

Figure.3

\section{Mathematical Expression of OFDM.}

In 1971 Weinstein and Ebert put forward that using discrete Fourier transform to achieve frequency division multiplexing parallel data transmission. Consider a data sequence:d0,d1,...dn-1 Each data is a complex number $(\mathrm{dn}=\mathrm{an}+\mathrm{bnj}$ is the output of the digital modulator, such as QAM,PSK and so on ), Suppose the sequence is made discrete inverse Fourier transform to obtain a sequence of $\mathrm{N}$ complex numbers $\mathrm{Sm}$.

$$
S_{m}=\sum_{n=0}^{N-1} d_{n} \exp \left(j 2 \pi \frac{m n}{N}\right)=\sum_{n=0}^{N-1} d_{n} \exp \left(j 2 \pi f_{n} t_{m}\right) \quad m=0,1,2 \cdots N-1
$$

Where : $f_{n}=\frac{n}{N T_{S}}, t_{m}=m T_{S}$.

$\mathrm{T}_{\mathrm{S}}$ represent the symbol interval of the original sequence. The real part of the formula in the sequence put in a low-pass filter and let the interval between the sign equals $T_{S}$ and then the signal of OFDM is obtained.

$$
y(t)=\operatorname{Re}\left\{\sum_{n=0}^{N-1} d_{n} \exp \left(j 2 \pi \frac{n}{T} t\right)\right\}=\sum_{n=0}^{N-1} a_{n} \cos \left(2 \pi f_{n} t+b_{n} 2 \pi f_{n} t\right) 0 \leq t \leq T
$$


In the formula the length of the OFDM symbol is $T$, the sub-carrier spacing is $1 / T$, and the system includes $\mathrm{N}$ orthogonal sub-carriers. In a cycle, during the different sub-carriers, they are orthogonally:

$$
\int_{0}^{T} \cos 2 \pi f_{n} \tau \cos 2 \pi f_{m} \tau d \tau=0 m \neq n
$$

And in a general OFDM system, to make the signal power before and after the IFFT and the FFT remains constant, the following definition of the DFT as:

$$
\begin{aligned}
& D F T: X(k)=\frac{1}{\sqrt{N}} \sum_{N=0}^{N-1} x(n) \exp \left(-j \frac{2 \pi k n}{N}\right) \\
& 0 \leq k \leq N-1 \\
& I D F T: x(n)=\frac{1}{\sqrt{N}} \sum_{N=0}^{N-1} X(k) \exp \left(j \frac{2 \pi k n}{N}\right) \\
& 0 \leq n \leq N-1
\end{aligned}
$$

In the following simulations, we will use the definition of the formula (7) and formula (8).

\section{Simulation and Analysis}

MATLAB simulation analysis is according to figure.3 and formula (7) and formula (8).First of all, Do code mapping In the frequency domain, and then, After serial to parallel conversion and IFFT transform, Take the signals transformed into the time domain, which is equivalent to the modulation to each orthogonal sub-carriers. At Last, complete orthogonal frequency division multiplexing. According to the need, add the guard space that is cyclic prefix, and also window function in the OFDM signal to reduce the power of out of band signals. The receiver to complete a contrary process of the sender.FFT demodulates the time domain signal to the frequency domain, output signal after it balanced, judged and decoded.

BER performance is compared in AWGN and multipath interference (with OFDM and non OFDM).

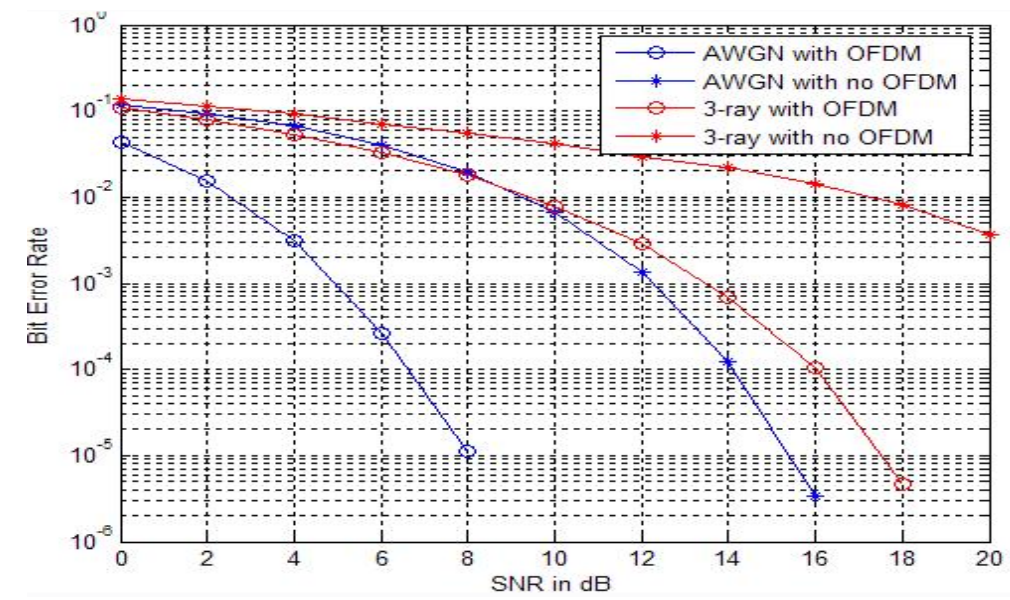

Figure.4

The results of BER curves comparing shown in Figure.4 curve from bottom respectively: AWGN channel with OFDM, AWGN channel without OFDM, AWGN + 3-path channel with OFDM, AWGN + 3-path channel without OFDM, We can see from Figure.4 the error rate is higher and higher, the performance of the system to reduce in turn. The System used OFDM modulation, can reduce the effects of AWGN and multipath interference, the BRE performance is improved significantly. The BER performance of system under the multipath interference than only under AWGN interference is much worse. This is mainly due to inter-symbol interference caused by multipath delay affects the BER performance of the system. According to the simulation analysis can be concluded that the OFDM technology to enhance the performance of LVPLC system has a significant role. 


\section{Conclusions}

Through the study of low voltage power line carrier communication system based on OFDM , the LVPLC system without laying new lines and its communication speed, strong anti-interference ability, high communication accuracy, and other advantages, will bring new vitality to our intelligent home life.

\section{Acknowledgements}

This work was financially supported by the Innovation Program of Shanghai Municipal Education Commission (12zz191) and NSFC 51405303.

\section{References}

[1] M. Gotz, M. Rapp, K. Dostert. Power Line Channel Characteristics and Their Efleet On Communication System Design[J]. IEEE Communications Magazine, 42(4):78-76(2004).

[2] Jose Antonio Cortes, Luis Diez. Analysis of the Indoor Broadband Power Line Noise Scenario[C].IEEE Transactions on Electromagnetic Compatibility. 849-858(2010).

[3] Chu He. Low Voltage Power Line Communication Module of FPGA Implementation [D]. Harbin: Harbin university of science and technology (2009).

[4] Zhang Youbin, Chen Shijie, He Haibo. Modeling of Low voltage Power Line High Frequency Communication Channel [J].WangFan Data. 26(23): 1-5(2002).

[5] Geng Kang, Li Yonghui. Analysis of Low Voltage Power Line Communication Channel Characteristic and Model [J]. Power System Communication ,4:19-24(2004).

[6] Information on http://www. wikipedia.org. 